\title{
Crack Identification of Infrared Thermal Imaging Steel Sheet Based on Convolutional Neural Network
}

\author{
Wang Wei ${ }^{1}$, Li Qing ${ }^{1, ~ *}$ \\ ${ }^{1}$ National and local joint engineering laboratories for disaster monitoring technologies and instruments, China Jiliang University, Hangzhou \\ 310018, China
}

\begin{abstract}
Aiming at the low efficiency and poor anti-interference ability of traditional non-destructive testing technology in steel plate crack detection, a crack recognition method based on convolutional neural network for infrared thermal imager is proposed. Firstly, a rolling electric heating rod is developed as a thermal excitation source, and a new excitation method was used to thermally excite the surface to be inspected. Then, according to the principle of abnormal temperature generated during the heat transfer process, the temperature of the detected surface is analyzed. It is concluded that the temperature gradient on both sides of the crack is always the largest. Finally, the infrared thermal image after thermal excitation is collected as a training sample, and a convolutional neural network is built to train the sample. Experiments show that the convolutional neural network model can accurately identify the infrared image cracks. The detection efficiency is high and the robustness is strong. And the recognition accuracy on the test set reaches $96.82 \%$.
\end{abstract}

\section{Introduction}

As the main transportation tool for dangerous chemicals, tank trucks will directly affect the safety of transportation throughout the railway. Due to the defects of the inner wall steel plate during the manufacturing process, the stress concentration and the long-term fatigue load, cracks will occur in the inner wall of the tank. If the crack is not found in time, it will become a huge safety hazard, so the crack of the steel plate of the tank car detection is especially important.

At present, the detection methods of cracks in tank steel plates mainly include magnetic powder detection, ultrasonic detection and magnetic flux leakage detection. The steps of magnetic particle detection are complicated and the detection efficiency is low [1]. The surface of the ultrasonic inspection requires a coupling agent, and the shape, orientation and position of the defect often affect the detection result [2]. The magnetic flux leakage detection equipment is expensive [3], and the detection cost is high. Dark, the inner wall of the tank is often accompanied by debris such as welding slag, rust and oil oxide layer [4]. For the case of tank car body, the traditional detection method has low detection efficiency and poor anti-interference ability. If the cleaning is not clean, the detection result will have a great influence or even false detection.

Aiming at this situation, a crack detection method for tank steel plate based on infrared thermal imaging is proposed. The crack is judged by analyzing the temperature gradient between the regions in the thermal image. The acquired infrared image is sent to the convolutional neural network for training.

\section{Infrared thermal imaging}

\subsection{Detection method}

The current detection methods are mainly divided into reflection method and transmission method. The main difference is whether the excitation source is on the same side as the thermal imager. The principle of utilization is to judge the crack by analyzing the propagation process of heat in the vertical direction of the object. Because the energy radiated by the object collected by the infrared camera is mainly related to its temperature and emissivity, the surface of the tank wall is often accompanied by uneven surface emissivity, which will cause false "high and low temperature phenomena" in the thermal image [5], false high and low temperature is easy to cause misjudgment of cracks.

In order to solve the above problems, this paper proposes a new incentive method. When thermally excited from one side of the object, the steady state conditions of the object are destroyed. At this time, heat is

\footnotetext{
* Author to whom correspondence should be addressed; E-Mail: lq13306532957@163.com

Item Number : 2014QK198.
} 
diffused in the horizontal direction and the vertical direction. Due to heat transfer, when the heat diffused in the horizontal direction encounters a crack, heat is concentrated on one side of the crack, and the temperature difference between the two sides of the crack increases. The detection principle is shown in figure 1. By analyzing the abnormality of the temperature difference between the horizontal direction regions, the crack detection can avoid the interference caused by the uneven local heating and the uneven emissivity.

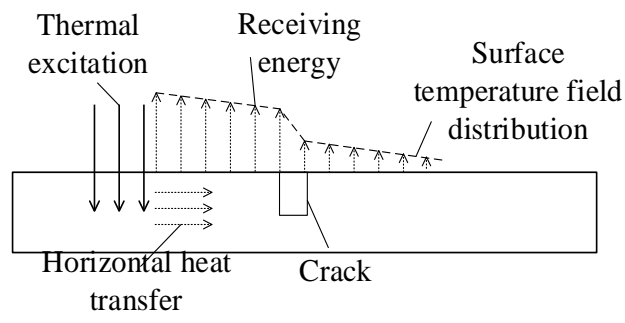

Figure 1. Horizontal heat conduction diagram

\subsection{Research on thermal excitation devices}

According to the proposed excitation method, a rolling electric heating rod is designed as a thermal excitation source. The mechanical structure is shown in figure 2 .

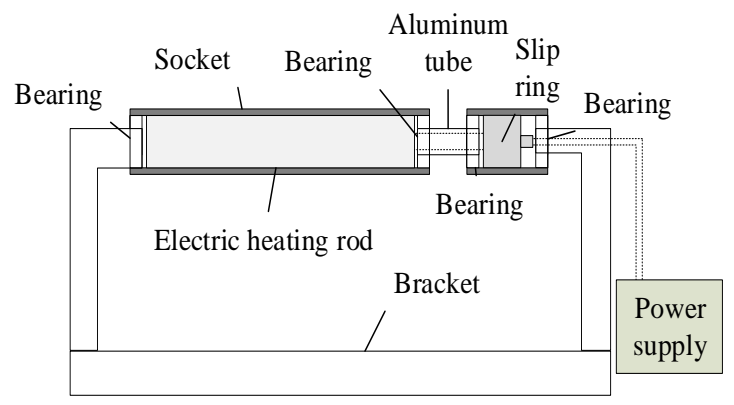

Figure 2. Rolling electric heating rod structure

It is mainly composed of electric heating rod and energized slip ring, stainless steel tube sleeve, bearing and thin aluminum tube. The electric heating rod is filled with insulating magnesium oxide crystal, and the heating wire is nickel-chromium wire. The power is $100 \mathrm{w}$, and the temperature can be quickly raised to 150 Celsius. Since the line at both ends of the heating rod is fixed, the electric heating rod may have a problem of wire winding during the rolling process, so the power-slip ring is connected to the wire end of the heating rod, and the highest temperature resistance of the energizing sliding ring is 80 degrees celsius. So separate the slip ring from the heating rod with an aluminum tube so that the temperature does not exceed its temperature resistance.

\subsection{Scheme design}

The zoned block excitation is performed by using the designed rolling heating rod, after continuous excitation, it continues to roll forward to the next detection area for excitation. In the process of excitation, heat is transmitted in the horizontal direction, the temperature is abnormal when transmitted to the crack, and the difference is not significantly different from the non-cracked area, and the infrared thermal image is recorded in real time. The crack is identified and located by analyzing the temperature variation curve and the infrared heat map anomaly area The design of the detection system is shown in figure 3 .

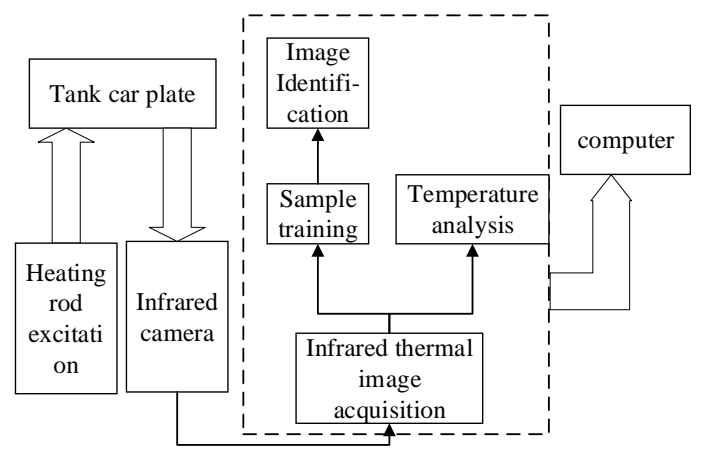

Figure 3. System plan design

\section{Convolutional Neural Networks}

Convolutional neural network, as a model structure of deep learning, is a deep learning multilayer perceptron specially designed to recognize two-dimensional shapes. In structure, it is mainly composed of single or multiple convolutional layers and pooling layers. Due to its weight sharing and local sensation, it has the ability to extract features more efficiently than other deep learning models [6].

Generally, the convolution process is as shown in equation (1):

$$
x_{j}^{l}=f\left(\sum_{i \in M_{j}} x_{i}^{l-1} * w_{i j}^{l}+b^{l}\right)
$$

In the formula: $l$ represents the number of layers, $w$ represents the convolution kernel, $M_{j}$ represents a choice of the input feature map, and $b$ represents the offset.

Max-pooling is used in the pooling layer, and the process is shown by formula (2):

$$
x_{j}^{l}=\operatorname{down}\left(x_{j}^{l-1}\right)
$$

\subsection{Structural parameters}

The main structure of the convolutional neural network is shown in figure 4.The convolutional neural network used in this paper consists of seven layers.

The original image is normalized as a network input; the first convolution layer uses 32 convolution kernels; the second convolution layer uses 64 convolution kernels; the third convolution layer uses 32 convolution kernels. The pooling layer is downsampled by a $2 \times 2$ convolution kernel; after that, the network passes through two layers of fully connected layers, and the output is 2 neurons. 


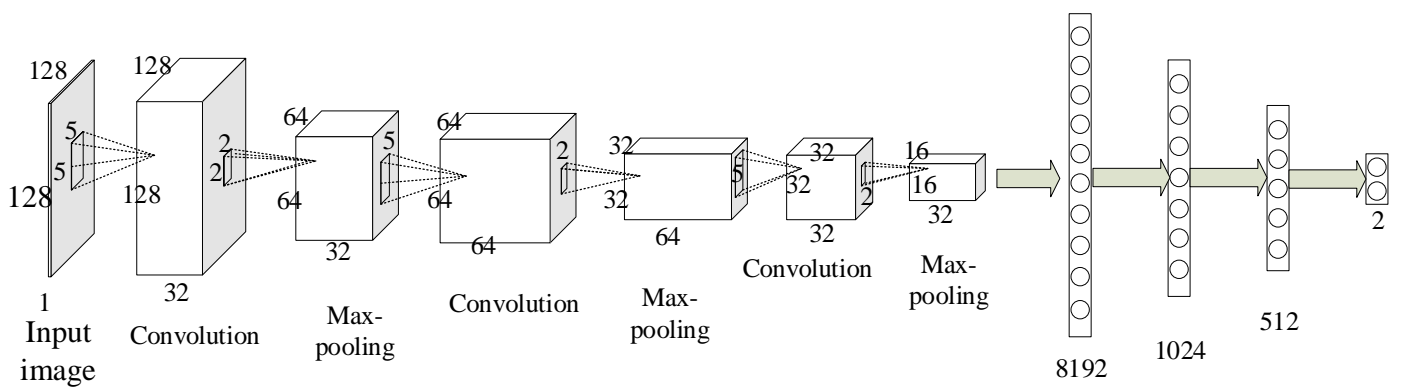

Figure.4 Convolutional neural network structure

\subsection{Selection of activation function and loss function}

The nonlinear transformation layer is an operation of processing the upper layer feature image with a nonlinear excitation function. It can smoothly classify images and make the neural network more expressive, which can simulate more complex models. Relu activation function is shown in Equation 3:

$$
\operatorname{relu}(z)= \begin{cases}0 & z<0 \\ z & z>=0\end{cases}
$$

The output layer activation function uses the Softmax function, and the function expression is:

$$
\hat{y}^{(i)}=\frac{e^{\left(z_{i}\right)}}{\sum_{j=1}^{k} e^{\left(z_{j}\right)}}
$$

In the formula: $\mathrm{z}$ represents the neuron linear house result of the output layer, and $\mathrm{k}$ represents the number of neurons in the output layer.

The loss function uses a cross entropy cost function, and the function expression is:

$$
J(w, b)=\frac{1}{m}\left[\sum_{i=1}^{m}\left(-y^{(i)} \log \hat{y}^{(i)}-\left(1-y^{(i)}\right) \log \left(1-\hat{y}^{(i)}\right)\right)\right](5)
$$

In the formula: $m$ represents the number of samples, and $y^{(i)}$ represents the i-th sample tag value, $\hat{y}^{(i)}$ represents the neural network output value of the $\mathrm{i}$-th sample.

\subsection{Optimizer selection}

The Adam algorithm is a learning rate adaptive optimization algorithm [7]. First, in Adam, momentum directly incorporates an estimate of the gradient first-order moment. Second, Adam includes an offset correction that corrects the first-order moment and second-order from the origin initialization. Estimation of the moment. The specific algorithm implementation is as follows.

$$
\begin{gathered}
V_{d w}=\beta_{1} V_{d w}+\left(1-\beta_{1}\right) d w \\
S_{d w}=\beta_{2} S_{d w}+\left(1-\beta_{2}\right)(d w)^{2} \\
V_{d w}^{\text {corrected }}=\frac{V_{d w}}{1-\beta_{1}^{t}} \\
S_{d w}^{\text {corrected }}=\frac{S_{d w}}{1-\beta_{2}^{t}}
\end{gathered}
$$

$$
w:=w-\frac{\alpha V_{d w}^{\text {corrected }}}{\sqrt{S_{d w}^{\text {corrected }}}+\varepsilon}
$$

In formula (6-10): $V_{d w}$ is the first-order moment estimate; $S_{d w}$ is the second-order moment estimate, initialized to 0 and then updated; dw is the differential of $\mathrm{w} ; V_{d w}^{\text {corrected }}$ and $S_{d w}^{\text {corrected }}$ are the correction of first-order and second-order moments respectively; $\alpha$ is the learning rate; $\mathrm{w}$ is the weight parameter and $\mathrm{w}$ : is the updated weight parameter.

\section{Experimental analysis}

\subsection{System components}

The experimental system is mainly composed of a rolling electric heating rod, an infrared thermal imager, a computer, and a workpiece to be inspected. The infrared camera used in the experiment is FLIR A315. The thermal excitation source is the rolling electric heating rod designed in this paper, and the physical diagram is shown in figure 5 .

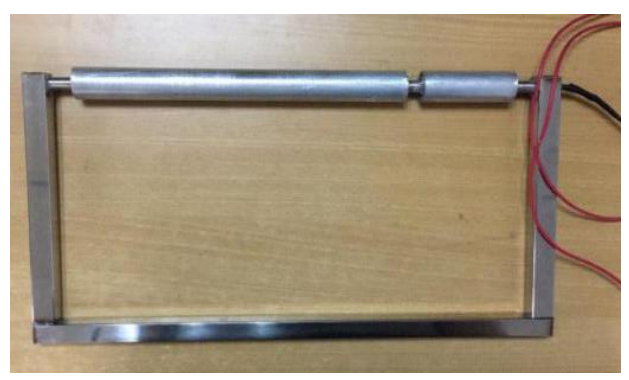

Figure.5 Rolling electric heating rod physical map

The test piece is selected as a test piece with cracks and severe surface corrosion. The dimension is $20 \mathrm{~cm}$ in length, $10 \mathrm{~cm}$ in width and $0.5 \mathrm{~cm}$ in thickness. The surface is closed with sand and rust, and the surface emissivity is extremely uneven. This practice simulates the actual condition of the inner wall of the tank. The front view and the crack detail are shown in figure 6 . The crack size is $2 \mathrm{~cm}$ in length, $0.5 \mathrm{~mm}$ in width and $3 \mathrm{~mm}$ in depth. 


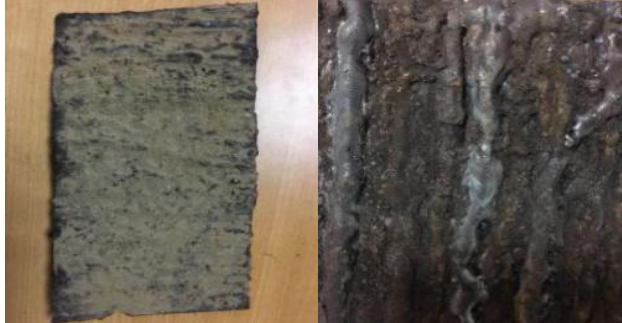

Figure.6 Steel plate test piece

\subsection{Temperature analysis}

The rolling electric heating rod is closely attached to the surface of the steel plate test piece. The heating rod is kept stationary, and it is continuously excited for $100 \mathrm{~s}$ at 150 Celsius, and cooled for $50 \mathrm{~s}$. The temperature changes of the four points of $\mathrm{Sp} 1, \mathrm{Sp} 2, \mathrm{Sp} 3$, and $\mathrm{Sp} 4$ are recorded separately. The excitation process picture and the four-point position are shown in figure 7.

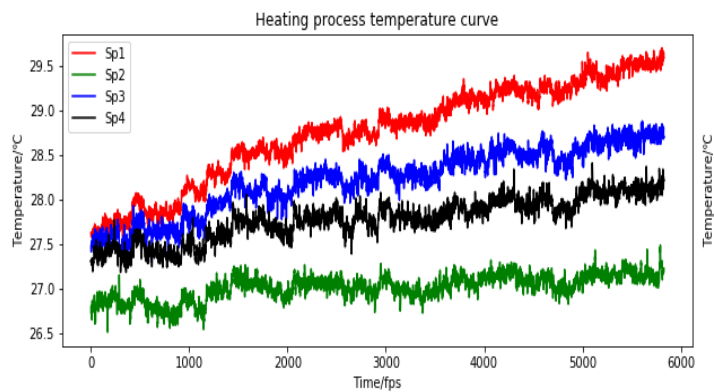

(a) Cooling process

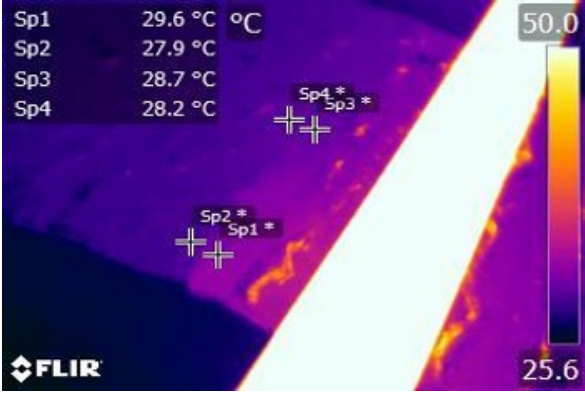

Figure.7 Incentive process picture

The excitation process and the temperature change after excitation are shown in (a) and (b) of figure 8. It can be seen that the temperature at Sp1 is always the largest and the fastest rise, and the temperature at $\mathrm{Sp} 2$ does not change much during the heating process; Sp3 and Sp4 are compared with Sp1. The temperature rises more slowly. The temperature difference between Sp1 and Sp2 increases rapidly, and the temperature difference between Sp3 and Sp4 is basically small, and the temperature difference between $\mathrm{Sp} 1$ and $\mathrm{Sp} 2$ is always much larger than the temperature difference between Sp3 and Sp4.

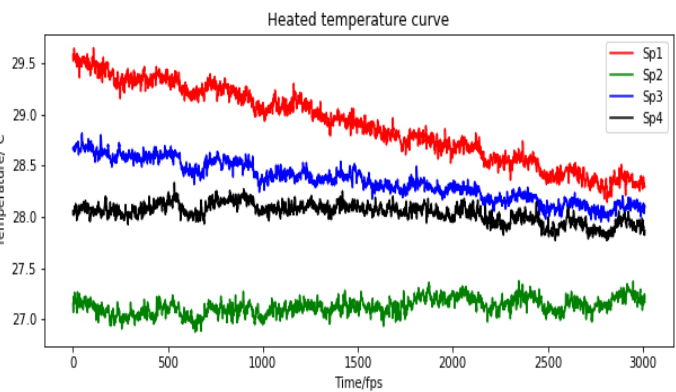

(b) Heating process

Figure.8 Temperature change in time

\subsection{Image Recognition and Verification}

In the infrared thermal image, the color difference is the gradient characteristic of the image. The temperature analysis experiments show that in the infrared thermal image with crack, the temperature difference between crack and non-crack region is larger, and the image gradient characteristics are more obvious.

Rolling electric heating rods were used to perform rolling thermal excitation on crack and non-crack areas of multiple steel plates, and infrared thermal images were collected in real time by infrared camera. The original image collected has a total of 1000 images, and the image is horizontally mirrored, cut, twisted, etc., and the image is expanded to 3000 images, of which 1500 images contain cracks and 1500 images do not contain cracks. The 3000 images were divided into training sets and test sets according to a 4:1 ratio. The training set was 2400 images and the test set was 600 images. The output label is marked in one-hot encoding format. Sample example is shown in figure 9.

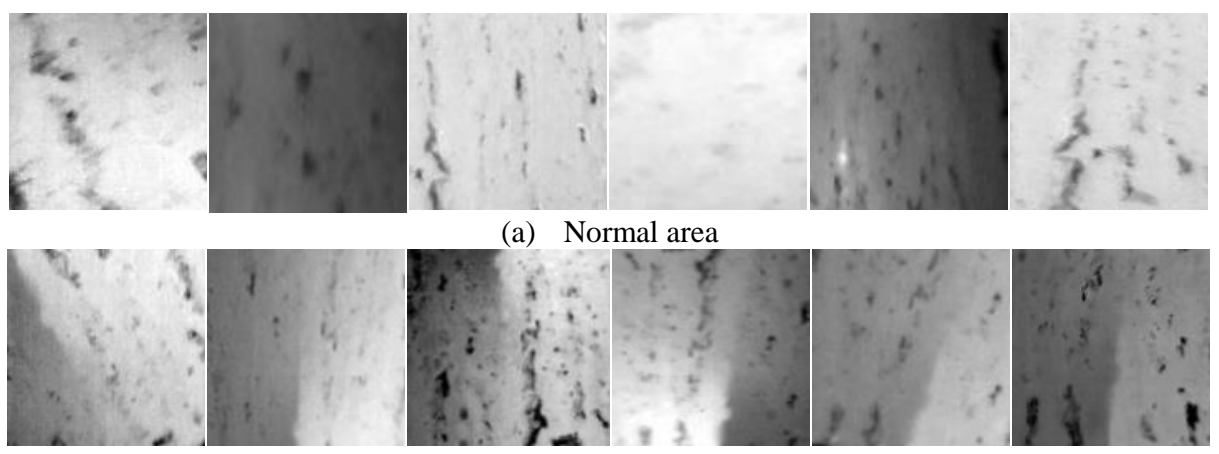

(b) Cracks area

Figure.9 Sample example 
The experiment is conducted in batches. Finally, all the parameter debugging results are as follows: the size of the batch-size is set to 64 ; the learning rate attenuation strategy is used for learning, the initial learning rate is set to $1 \mathrm{e}-3$, and the attenuation is 0.99 times per iteration; The weight $\mathrm{w}$ initialization method is initialized with a truncated normal distribution, and the offset $\mathrm{b}$ is all set to 0.01 ; in the Adam algorithm, $\beta 1$ is set to $0.9, \beta 2$ is set to
0.999 , and set to $1 \mathrm{e}-8$; the random inactivation probability of the Dropout neuron is set to $75 \%$.

The curve of the model loss value with the number of iterations during the training process is shown in figure 10 , the pre-test of the training set and the test set. And the curve of the measurement accuracy with the number of iterations is shown in figure 11 .

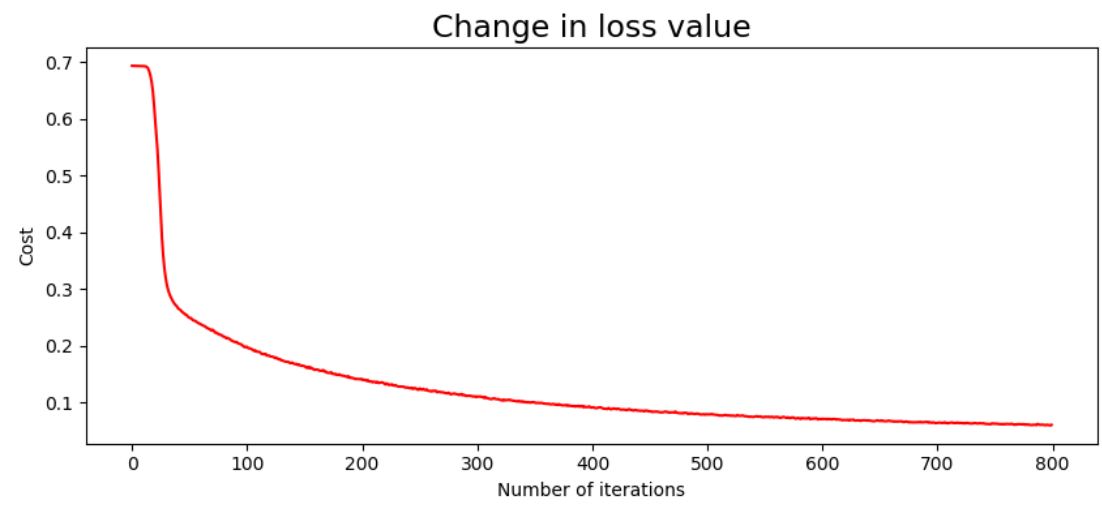

Figure.10 Loss value change curve

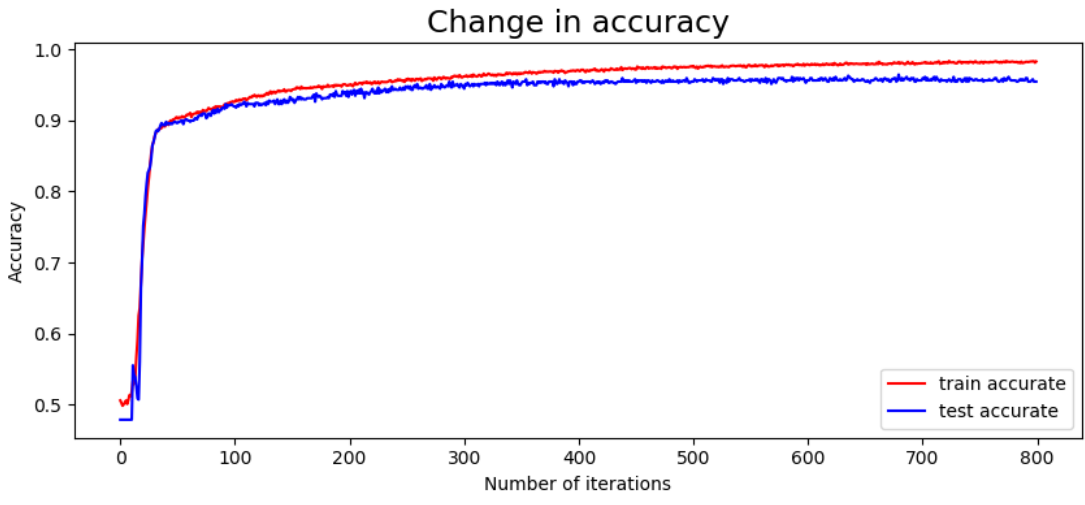

Figure.11 Accuracy change curve

The experimental results show that the loss value of the model decreases rapidly and the prediction accuracy increases rapidly 100 times before training. When the training is about 600 times, the model begins to converge. When the training reaches 800 times, the model loss value has stabilized. The prediction accuracy on sets and test sets remains stable. The final recognition accuracy on the test set reached $96.82 \%$.

\section{Conclusions}

This paper verifies the feasibility of automatic identification of cracks in tank steel plates based on infrared thermal imaging. Using the rolling electric heating rod combined with the new thermal excitation method, the surface of the test piece can be thermally and simply excited. By analyzing the temperature of the cracked area and the non-cracked area, the law of temperature change is obtained, and the temperature difference between the areas is changed. The situation is judged by the crack condition; the model is trained by the convolutional neural network, and the automatic identification of the crack is finally realized.

\section{References}

1. Qi Wenyang, Gao Shanglei, Zhang Boying. Nondestructive testing technology for in-service storage tanks. J. Petrochemical and Chemical Industry Standards and Quality; 33(11): 11 (2012)

2. Li Xingcai. Research on Nondestructive Testing Methods in Pressure Vessel Inspection. J. Engineering Research, (02): 116-117 (2017)

3. Zhang Wei, Cai Qingqing, Zhang Lei. Application of magnetic flux leakage detection technology in an oilfield in Xinjiang. J. Non-destructive Testing, 33(02): 48-51 (2011)

4. Zhang Xingzheng, Song Longjun, Gao Zhaofu. Causes and protection of surface corrosion of liquefied petroleum gas tanks in tanks. J. Heilongjiang Science and Technology Information, (24): 19 (2007)

5. Bai Qiuju. Mechanism of thermal emissivity of material surface in thermal imaging detection and its suppression method. D. (2016) 
6. Li H, Lin Z, Shen X. A convolutional neural network cascade for face detection. Proceedings of the IEEE Conference on Computer Vision and Pattern Recognition, CVPR, 5325-5334 (2015)

7. Ersan YAZAN, M. Fatih Talu. Comparison of the stochastic gradient descent based optimization techniques. IDAP, 1-5 (2017) 